Supporting Information for

\title{
Deformation Hysteresis of Electrohydrodynamic Patterning on Thin Polymer Film
}

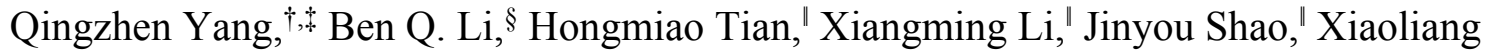

$$
\text { Chen," and Feng } \mathrm{Xu}^{*,+, *}
$$

†The Key Laboratory of Biomedical Information Engineering of Ministry of Education, School of Life Science and Technology, Xi'an Jiaotong University, Xi'an, Shaanxi 710049, P. R. China

Bioinspired Engineering and Biomechanics Center (BEBC), Xi'an Jiaotong University, Xi'an, Shaanxi 710049, P. R. China

${ }^{\S}$ Department of Mechanical Engineering, University of Michigan-Dearborn, Dearborn, Michigan 48128, United States

'State Key Laboratory for Manufacturing Systems Engineering, Xi'an Jiaotong University, Xi'an, Shaanxi 710049, P. R. China

*Corresponding author. Email: fengxu@mail.xjtu.edu.cn

\section{MATHEMATICAL FORMULATION}

We take the configuration in Figure 1 as an example. Both the polymer and air are considered as dielectric. For such materials, the external electric field applied upon it would polarize the molecules, and the molecular dipoles so induced in return modifies the electric field. Eventually the electric field is described by the Gauss Law $\nabla \cdot\left(\varepsilon_{0} \varepsilon_{r} E\right)=q^{e},{ }^{1}$ where $\varepsilon_{r}$ is the dielectric constant of the material, $\boldsymbol{E}$ the electric field intensity and $q^{e}$ free charge density. For an initially electroneutral dielectric material, the free charge would always remain zero $\left(q^{e}=0\right)$. Besides, for the electrohydrodynamic patterning process the electrical current is small, and thus the magnetic field is small enough to be neglected. Besides, the curl of the electric field is approximately zero $(\nabla \times E=0)$, which allows us to write $E=-\nabla V$ (where $V$ denotes the electric potential). Thus the governing equation of electric field for dielectric materials can be simplified to the Laplace equation, ${ }^{2-4}$ 


$$
\nabla^{2} V_{i}=0 \quad \in \Omega_{i} \mid i=1,2
$$

this equation governs both the air and polymer where subscript $i$ refers to the $i$ th domain $(i=1$ for air, $=2$ for polymer).

The boundary conditions are needed to enable the solution of electric field, which express as follows,

$$
\begin{aligned}
& V_{1}=U \quad \in\left\{\partial \Omega^{1} \mid z=d(x, y)\right\} \\
& \frac{\partial V_{i}}{\partial n}=0 \quad \in\left\{\partial \Omega^{1} \cup \partial \Omega^{2}\left|x=0 \vee x=x_{0} \vee y=0 \vee y=y_{0}\right| i=1,2\right\} \\
& V_{2}=0 \quad \in\left\{\partial \Omega^{2} \mid z=0\right\} \\
& V_{1}=V_{2}, \quad \varepsilon_{1} \frac{\partial V_{1}}{\partial n}=\varepsilon_{2} \frac{\partial V_{2}}{\partial n} \quad \in\left\{\partial \Omega\left|\partial \Omega:=\Omega_{1} \cap \Omega_{2}\right| z=h(x, y)\right\}
\end{aligned}
$$

where $\partial \Omega$ is the boundary of the domain $\Omega$ and $\mathbf{n}$ is the surface normal of the boundary. Physically, the boundary conditions indicate the system is symmetric about the lateral surfaces $x=0, x=x_{0}, y=0$ and $y=y_{0}$. At the top $z=d(x, y)$ and bottom surface $z=0$, the electric potential is prescribed. As for the polymer/air interface, the electric potential and displacement are continuous. ${ }^{1}$

Instead of accurately solving the governing equation (1) and the associated boundary conditions. We pursue an approximate solution in this study. The periodic length $l_{0}$ is assumed to be much larger than the initial film thickness $h_{0}$, thus a "long-wave approximation" can be used to simplify the governing equation. At the polymer/air interface, the electrical displacement in the tangential direction can be ignored $E_{l}=0$ and that in the normal direction expresses,

$$
D_{n}=\frac{\varepsilon_{1} \varepsilon_{2} U}{\varepsilon_{1} h+\varepsilon_{2}(d-h)}
$$

this equation would be useful when solving the polymer/air interfacial deformation. 
The next step is to derive the governing equations of interfacial deformation $h(x, y)$. This study focuses on the equilibrium state of electrohydrodynamic patterning. At such a state, the total force is balanced across the interface,

$$
\sigma_{i, j}^{(1)} \cdot \vec{n}-\sigma_{i, j}^{(2)} \cdot \vec{n}=\gamma\left(\nabla_{s} \cdot \vec{n}\right) \vec{n}
$$

where $\gamma$ is the surface tension coefficient, $\nabla_{s}=\nabla-\vec{n}(\vec{n} \bullet \nabla)$ the surface divergence operator. ${ }^{5} \sigma_{i, j}^{(k)}$ represents the stress of the fluid $k(k=1$ for the air, 2 for the polymer), and it expresses as the sum of the hydrostatic pressure, viscous stress tensor and electrical stress tensor,

$$
\sigma_{i j}^{(k)}=-p^{(k)} \delta+\mu^{(k)}\left(\frac{\partial u_{i}}{\partial x_{j}}+\frac{\partial u_{j}}{\partial x_{i}}\right)+\left[\varepsilon_{0} \varepsilon_{r} E^{(k)} E^{(k)}-\frac{1}{2} \varepsilon_{0} \varepsilon_{r} E^{(k)} \cdot E^{(k)} \delta\right]
$$

where $p^{(k)}$ is the hydrostatic pressure, $\mu^{(k)}$ the viscosity, $\boldsymbol{E}$ the electric field strength, $x_{1}, x_{2}$ and $x_{3}$ represent the $x, y$, and $z$ direction, and $u_{i}, u_{j}$ the fluid velocity in $x_{i}, x_{j}$ direction. Since the equilibrium state problem is under consideration here, the viscous contribution $u^{(k)}, v^{(k)}$ are null as there is no fluid flow at equilibrium state; otherwise the interface would keep evolving due to the fluid motion. The governing equation (3) can be decomposed in $x, y$ and $z$ directions to three equations. It was found that the equations in $x$ and $y$ directions could be satisfied by themselves, leaving the equation in $z$ direction,

$$
\underbrace{\frac{\nabla^{2} h}{\left[1+(\nabla h)^{2}\right]^{2 / 3}}+P_{g a g}}_{L}+\underbrace{\frac{1}{2}\left[\varepsilon_{0} D_{n}{ }^{2}\left(\frac{1}{\varepsilon_{1}}-\frac{1}{\varepsilon_{2}}\right)+\varepsilon_{0} E_{t}{ }^{2}\left(\varepsilon_{2}-\varepsilon_{1}\right)\right]}_{T}+\underbrace{\left[\frac{A_{L}}{6 \pi h^{3}}-\frac{A_{U}}{6 \pi(d-h)^{3}}\right]}_{M}=0
$$

where $h(x, y)$ denotes the polymer/air interface measured from the substrate, $\nabla^{2}=\frac{\partial^{2}}{\partial x^{2}}+\frac{\partial^{2}}{\partial y^{2}}$ the Laplacian operator, $P_{g a g}=P^{(2)}-P^{(1)}$ the gauge pressure, $D_{n}$ the normal component of the electric displacement and $E_{t}$ the tangential component of the electric field. It is noted that an extra term $M$ is added here, which represents the Lifshitz-van der Waals (LW) force. ${ }^{6-7}$ Essentially, it indicates a repulsive force exists between the polymer-template and polymer-substrate. In experiments, this Lifshitz-van der Waals force becomes significant when the polymer film gets infinitely close to (i.e., touches) the template or the substrate. $A_{L}$ and $A_{U}$ represent the effective Hamaker constant for lower and upper electrode the value of which depend on the materials used for electrodes. With this term considered, we can successfully model the attachment and 
de-attachment of the polymer film with the template, which has been observed in experiments. ${ }^{8}$ For convenience, we use $L$ to denote the Laplace pressure, $T$ the electrostatic pressure, and $M$ the disjoing pressure. Substituting the equation (2) into (4), one would have the governing equation,

$$
\nabla^{2} h+P_{\text {gag }}+\frac{1}{2} \varepsilon_{0}\left(\frac{1}{\varepsilon_{1}}-\frac{1}{\varepsilon_{2}}\right)\left[\frac{\varepsilon_{1} \varepsilon_{2} U}{\varepsilon_{1} h+\varepsilon_{2}(d-h)}\right]^{2}+\left[\frac{A_{L}}{6 \pi h^{3}}-\frac{A_{U}}{6 \pi(d-h)^{3}}\right]=0
$$

Again the "long-wave approximation" is used here, which leads to $\nabla h \ll 1$. To solve such an equation, the appropriate boundary conditions are needed. In this study, we employ the Neumann boundary conditions at the lateral surfaces (see Figure 1b),

$$
\left.\frac{d h}{d x}\right|_{x=0}=\left.\frac{d h}{d x}\right|_{x=x_{0}}=\left.\frac{d h}{d y}\right|_{y=0}=\left.\frac{d h}{d y}\right|_{y=y_{0}}=0
$$

Besides, the constant $P_{\text {gag }}$ is still unknown in equation (5). An extra equation is needed to obtain $P_{g a g}$, here we take the mass conservation equation,

$$
\int_{0}^{y_{0}} \int_{0}^{x_{0}} h(x) d x d y=V_{0}
$$

with $V_{0}$ being the polymer film's initial volume. For the above governing equation (5) and the associated boundary conditions (6) and (7), usually the parameters $\sigma, \varepsilon_{1}, \varepsilon_{2}, A_{L}, A_{U}$ and the morphology of the template $d(x, y)$ are known for the system. Once the external voltage $U$ are prescribed, the polymer/air interface $h(x, y)$ can be resolved.

Computational Methodology. For convenience, the governing equation (5) is written as a function $G(h, U)=0$. To resolve it we used a finite difference method for a numerical solution. The aim is to get the free surface deformation $h$ for a preset value of $U$. Calculation begins with an estimated interface deformation, then it is substitute into equation (5) and starts iterating until it gets converged within a preset tolerance. Howbeit for such a highly nonlinear equation it would encounter a singularity problem at certain value of $U .{ }^{9}$ Thus we modified the algorithm by adopting the Keller's Pseudo-Arclength Continuation method ${ }^{10-12}$ and taking the $U$ as an arclength parametrization to help continue past turning points. Here a brief introduction is given for the implementation. Suppose two solutions of the function are known as $G\left(h_{0}, U_{0}\right)=0$ and $G\left(h_{1}, U_{1}\right)=0$. Then the next solution could be predicted by solving the following equations, 


$$
\left\{\begin{array}{l}
G\left(h_{2}, U_{2}\right)=0 \\
\left(h_{2}-h_{1}\right) \cdot\left(h_{1}-h_{0}\right)+\left(U_{2}-U_{1}\right)\left(U_{1}-U_{0}\right)=\Delta s
\end{array}\right.
$$

where $h_{2}$ is the interface deformation under the external voltage $U_{2} . \Delta s$ is the step length which controls the distance of two adjacent solutions $h_{1}, h_{2}$. The aforementioned scheme (i.e., finite difference method together with iteration) is capable to solve equation (8). This procedure repeats itself and a family of surfaces $h(x, y)$ with the corresponding external voltage $U$ are thus obtained.

\section{References}

(1) Jackson, J. D., Classical Electrodynamics. Wiley: 1999.

(2) Tomar, G.; Gerlach, D.; Biswas, G.; Alleborn, N.; Sharma, A.; Durst, F.; Welch, S. W. J.; Delgado, A., Two-Phase Electrohydrodynamic Simulations Using a Volume-of-Fluid Approach. J. Comput. Phys. 2007, 227 (2), 1267-1285.

(3) Hua, J. S.; Lim, L. K.; Wang, C. H., Numerical Simulation of Deformation/Motion of a Drop Suspended in Viscous Liquids Under Influence of Steady Electric Fields. Phys. Fluids 2008, 20 (11), 113302.

(4) Lin, Y.; Skjetne, P.; Carlson, A., A Phase Field Model for Multiphase Electrohydrodynamic Flow. Int. J. Multiphase Flow 2012, 45, 1-11.

(5) Weatherburn, C. E., Differential Geometry of Three Dimensions. Cambridge University Press: London: 1972.

(6) Wu, N.; Pease, L. F.; Russel, W. B., Electric-field-induced Patterns in Thin Polymer Films: Weakly Nonlinear and Fully Nonlinear Evolution. Langmuir 2005, 21 (26), 12290-12302.

(7) Wu, N.; Russel, W. B., Dynamics of the Formation of Polymeric Microstructures Induced by Electrohydrodynamic Instability. Appl. Phys. Lett. 2005, 86 (24), 241912.

(8) Heier, J.; Groenewold, J.; Steiner, U., Pattern Formation in Thin Polymer Films by Spatially Modulated Electric Fields. Soft Matter 2009, 5 (20), 3997-4005.

(9) Yang, Q.; Li, B. Q.; Ding, Y.; Shao, J., Steady State of Electrohydrodynamic Patterning of Micro/Nanostructures on Thin Polymer Films. Ind. Eng. Chem. Res. 2014, 53 (32), 12720- 
12728.

(10) Keller, H. B., Numerical Solution of Bifurcation and Nonlinear Eigenvalue Problems. Applications of bifurcation theory 1977, 359-384.

(11) Keller, H. B., Lectures on Numerical Methods in Bifurcation Problems. Springer: 1987.

(12) Spence, A., and Graham, I. G., Numerical Methods for Bifurcation Problems. Springer: Berlin Heidelberg: 1999. 